\title{
Allergic rhinitis is associated with poor asthma control in children with asthma
}

\author{
Eric P de Groot, ${ }^{1}$ Anke Nijkamp, ${ }^{1}$ Eric J Duiverman, ${ }^{2}$ Paul L P Brand ${ }^{1,3}$
}

${ }^{1}$ Princess Amalia Children's Clinic, Isala klinieken, Zwolle, The Netherlands

Beatrix Children's Hospital, University Medical Centre, Groningen, The Netherlands ${ }^{3}$ UMCG Postgraduate School of Medicine, University Medical Centre, Groningen, The Netherlands

\section{Correspondence to}

Eric P de Groot, Department of Pediatrics, Isala klinieken, Dokter van Heesweg 2, Zwolle 8025 AB, The Netherlands; e.p.de.groot@isala.nl

Received 28 September 2011 Accepted 9 December 2011 Published Online First 2 January 2012

\begin{abstract}
Background Asthma and allergic rhinitis are the two most common chronic disorders in childhood and adolescence. To date, no study has examined the impact of comorbid allergic rhinitis on asthma control in children. Objective To examine the prevalence of allergic rhinitis in children with asthma, and the impact of the disease and its treatment on asthma control.

Methods A cross-sectional survey in 203 children with asthma (5-18 years) using validated questionnaires on rhinitis symptoms (stuffy or runny nose outside a cold) and its treatment, and the paediatric Asthma Control Questionnaire (ACQ). Fraction of nitric oxide in exhaled air (FeNO) was measured with a Niox Mino analyser; total and specific lgE levels were assessed by the Immunocap system.
\end{abstract}

Results 157 children (76.2\%) had symptoms of allergic rhinitis but only 88 of these (56.1\%) had been diagnosed with the condition by a physician. ACO scores were worse in children with allergic rhinitis than in those without the condition $(p=0.012)$. An ACQ score $\geq 1.0$ (incomplete asthma control) was significantly more likely in children with allergic rhinitis than in those without (OR 2.74, 95\% Cl 1.28 to 5.91, $p=0.0081$ ), also after adjustment for FeNO levels and total serum lgE. After adjustment for nasal corticosteroid therapy, allergic rhinitis was no longer associated with incomplete asthma control (OR $0.72,95 \% \mathrm{Cl} 0.47$ to 1.12 , $\mathrm{p}=0.150$ ).

Conclusion Allergic rhinitis is common in children with asthma, and has a major impact on asthma control. The authors hypothesise that recognition and treatment of this condition with nasal corticosteroids may improve asthma control in children, but randomised clinical trials are needed to test this hypothesis.

\section{INTRODUCTION}

Asthma and allergic rhinitis (AR) are the two most common chronic disorders in childhood and adolescence, and their prevalence rates have doubled over the past decades. Current prevalence rates of AR in countries with a Western lifestyle may be as high as $40 \% .{ }^{1}$ It has long been recognised that AR and asthma commonly coexist, due to their similarities in anatomy, physiology and immunopathology. ${ }^{2}$ Surveys have shown that approximately $60-80 \%$ of children with asthma have symptoms of $\mathrm{AR}^{3-7}$

In adults, it has been shown that AR has a major impact on asthma morbidity, and that treatment of AR helps to improve asthma control. ${ }^{8}$ Despite its high prevalence, there are surprisingly few studies on the effects of treatment of AR in children. ${ }^{9}$

\section{Key messages}

What is the key question?

- The prevalence of allergic rhinitis in children with asthma, and the impact of the disease and its treatment on asthma control

What is the bottom line?

- Asthma and allergic rhinitis often coexist in children, and the presence of allergic rhinitis is associated with poorer asthma control

Why read on?

- This article analyses the relation of allergic rhinitis and asthma and the effect of allergic rhinitis therapy on asthma control.

Moreover, its impact on asthma in the paediatric age range has been poorly studied to date. A recent cross-sectional study in 404 French children with asthma showed symptoms of AR in $58 \%$ of the children, but no association with asthma severity was found. ${ }^{6}$ Conversely, a Japanese study of 150 children with asthma showed that exacerbations of upper and lower respiratory tract symptoms frequently coexisted, suggesting that AR does have an impact on asthma morbidity in children. ${ }^{5}$ To date, no study has examined the impact of comorbid AR on asthma control in children.

We designed this cross-sectional questionnaire based survey to determine the prevalence of AR in a Dutch population of children with asthma on inhaled corticosteroid (ICS) maintenance therapy, and the impact of AR on asthma control, level of lung function and exhaled nitric oxide.

\section{METHODS}

\section{Study population}

Between June 2008 and June 2009, consecutive patients aged 5-18years visiting the Princess Amalia asthma clinic for a scheduled follow-up visit were approached for participation in this crosssectional survey. Inclusion was continued over 1 year to allow all patients with asthma followed up at our clinic to participate in the survey.

\section{Inclusion and exclusion criteria}

Only patients fulfilling all of the following criteria were eligible for inclusion in the study: diagnosis of asthma, made by a paediatrician according to international standardised guidelines ${ }^{10}$; maintenance therapy with ICS for at least 3 months 
before the study; able to perform reproducible lung function testing; and proper understanding of Dutch language.

Patients with serious bronchopulmonary, neuromuscular or cardiovascular comorbidity were excluded.

\section{Questionnaire}

Symptoms of AR and level of asthma control were assessed by applying two standardised and validated questionnaires. The International Study of Asthma and Allergies in Childhood (ISAAC) rhinitis questionnaire was used to assess the presence of symptoms of $\mathrm{AR}^{11}$; asthma control was measured by the childhood Asthma Control Questionnaire (ACQ). ${ }^{12}{ }^{13}$ Both questionnaires have been validated for the Dutch language and have been used in earlier studies in the Netherlands. ${ }^{14} 15$

Patients aged 12 years or older filled out the questionnaires themselves; in children younger than 12 years, the parents completed the questionnaires.

AR (ever) was defined as a positive answer to the question: 'Has you child ever had a problem with sneezing, or a runny, or a blocked nose when he/she DID NOT have a cold or the flu?'

Aggregated ACO scores $<1.0$ were considered to indicate well controlled asthma. ${ }^{16}$

\section{Pulmonary function and nitric oxide measurement}

Immediately after filling out the questionnaires, complete expiratory flow-volume loops were recorded according to European Respiratory Society guidelines ${ }^{17}$ on a Jaeger MasterScreen (Jaeger, Houston, Texas, USA) by experienced and certified pulmonary function assistants. The forced expiratory volume in $1 \mathrm{~s}\left(\mathrm{FEV}_{1}\right)$ and forced vital capacity (FVC) were recorded, and expressed as a percentage of the predicted reference value. ${ }^{18}$ In addition, the fraction of nitric oxide in exhaled air (FeNO) was measured using the NIOX MINO device (Aerocrine, Solna, Sweden), according to standardised guidelines ${ }^{19}$ and expressed in parts per billion (ppb).

\section{Specific IgE to inhaled allergens}

Data on aeroallergen sensitisation were recorded from the chart if it had been obtained no longer than 2 years before the patient entered the study. The ImmunoCap system (Pharmacia Diagnostics, Uppsala, Sweden) was used for total and specific IgE assessments. As the detection limit of the assay was $0.35 \mathrm{kU} /$ litre, sensitisation was defined as a specific IgE concentration $\geq 0.35 \mathrm{kU} /$ litre. Screening tests were performed for the five major aeroallergens for children in the Netherlands (house dust mite, tree and grass pollen, cat and dog dander).

\section{Statistical analysis}

SPSS V.15.0 was used to analyse the data using t tests to compare means and $\chi^{2}$ tests to compare proportions. FeNO levels and ACQ scores were assessed by non-parametric methods because of their skewed distribution. Total IgE levels were log transformed before analysis. Multiple logistic regression analysis was performed to assess the relationship of dichotomised ACQ scores (well controlled vs incompletely controlled asthma, or ACQ $<$ or $\geq 1.0$ ) to allergic rhinitis after adjustment for potential confounders.

\section{RESULTS}

During the inclusion period, 206 patients fulfilling all inclusion criteria were approached for participation in the survey. None of these patients or their parents refused to participate. The clinical characteristics of the 206 patients are presented in table 1.
Table 1 Patient characteristics

\begin{tabular}{ll}
\hline Boys, number (\%) & $144(69.9 \%)$ \\
Age, mean (SD) & 10.4 years (3.7) \\
BMI, mean (SD) & $17.2(3.1)$ \\
Asthma maintenance therapy, n (\%) & $195(94.7)$ \\
ICS $\leq 250 \mu \mathrm{g} /$ day* & $143(73.3 \%)$ \\
ICS $251-500 \mu \mathrm{g} /$ day & $44(22.5 \%)$ \\
ICS $>500 \mu \mathrm{g} /$ day & $1(<1 \%)$ \\
Combination therapy (ICS+LABA) & $51(26.1 \%)$ \\
Allergic rhinitis therapy, $\mathrm{n}$ (\%) & $105(51 \%)$ \\
Nasal corticosteroids & $43(40.9 \%)$ \\
Oral antihistamines & $19(18.1 \%)$ \\
Montelukast & $4(3.8 \%)$ \\
Combination & $31(29.5 \%)$ \\
FVC, mean (SD) & $101.3 \%$ pred (12.0) \\
FEV ${ }_{1}$, mean (SD) & $100.3 \%$ pred (14.7) \\
FeNO, median (IQR) & 12.5 ppb (0-24) \\
IgE, median (IQR) & $247 \mathrm{kU} /$ litre (55-702) \\
\hline *Daily dose of fluticasone (or equivalent). & \\
BMl, body mass index (body weight (in kg) divided by height (in cm) \\
squared); ICS, inhaled corticosteroids; LABA, long-acting $\beta$ agonist; \\
FeNO, fraction of nitric oxide in exhaled air; FEV 1 , forced expiratory \\
volume in 1 s; FVC, forced vital capacity; pred, predicted.
\end{tabular}

\section{Prevalence of allergic rhinitis}

A total of 157 patients $(76.2 \%)$ had symptoms of AR, 144 of whom (91.7\%) reported complaints during the last 12 months (current AR).

AR was slightly more common in boys (112/144, $77.8 \%)$ than in girls $(45 / 62,72.6 \%)$, but the difference was not significant $(\mathrm{p}=0.40)$. A total of 88 patients $(56.1 \%$ of patients with rhinitis symptoms) had received a doctor's diagnosis of hay fever or AR. Symptoms of AR were considered to have an influence on daily activities in 102 children (64.9\%): $72 \%$ of these reported mild limitation, $25 \%$ moderate and $3 \%$ severe limitation of daily activities.

\section{Impact on asthma control}

In three patients, some answers to the ACQ were missing; complete ACQ data were obtained in 203 patients. An aggregated ACQ score $<1.0$, indicating well controlled asthma, was found in 128 children (63.1\%). Patients with AR were more likely to have an $\mathrm{ACO} \geq 1.0$ (indicating incomplete asthma control; 65 of 155 patients or $41.9 \%$ ) than those without AR (10 of $48,20.8 \%$, OR $2.74,95 \%$ CI 1.28 to $5.91, p=0.0081$ ). The distribution of ACQ scores in patients with and without AR is presented in figure 1 . The median (IOR) ACQ in patients with AR was higher $(0.67$, IOR $0.17-1.33)$ than in those without (0.33, IOR $0.04-0.67,95 \% \mathrm{CI}$ for difference, $\mathrm{p}=0.024)$. This was more pronounced in children $5-12$ years of age (median (IOR) in patients with AR $0.76(0.17-1.33)$ and $0.33(0-0.75)$ in those without, $p=0.052$ ) than in those $12-16$ years of age (median ACO in patients with AR $0.76(0.33-1.29)$, and in those without 0.50 (0.33-0.76), $\mathrm{p}=0.40)$.

\section{Relationship to lung function and FeNO levels}

Most patients with asthma had normal levels of $\mathrm{FEV}_{1}$ and $\mathrm{FVC}$ (table 1), and there were no differences in mean $\mathrm{FEV}_{1}$ or FVC between those with and without AR ( $p=0.50$ and 0.37 , respectively).

FeNO, however, was significantly higher in patients with AR (median $15 \mathrm{ppb}$, IOR 0-28 ppb) than in those without (median $7 \mathrm{ppb}$, IOR $0-12, \mathrm{p}=0.001$ ) (figure 2). No significant differences in FeNO levels were found between patients treated with nasal 


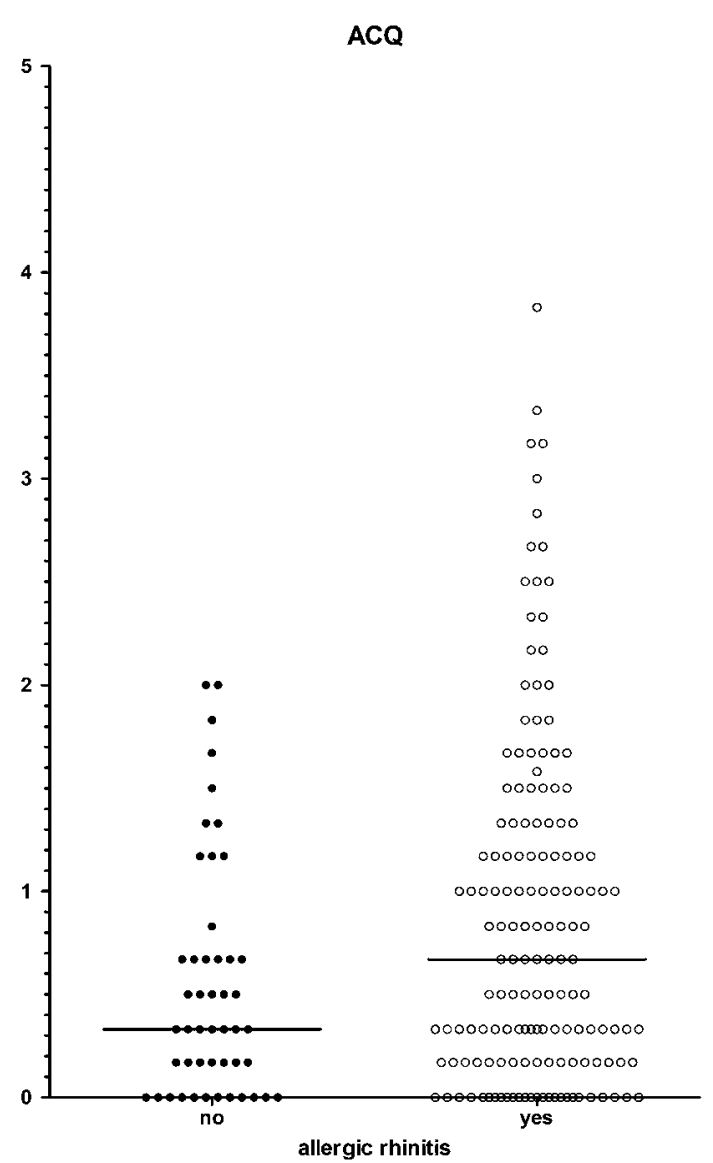

Figure 1 Distribution of Asthma Control Questionnaire (ACQ) scores in patients with asthma with and without allergic rhinitis; horizontal bars represent median levels. The ACO in patients with allergic rhinitis is significantly higher than in those without $(p=0.012)$, indicating poorer asthma control in patients with asthma and allergic rhinitis.

corticosteroids for $\mathrm{AR}$ and those who did not receive this treatment $(\mathrm{p}=0.816)$.

\section{IgE levels and aeroallergen sensitisation}

Data on total serum IgE levels and aeroallergen sensitisation obtained no more than 2 years before the survey were available in 130 patients (63.1\%). Total IgE levels were higher in patients with AR (geometric mean $680 \mathrm{kU} /$ litre) than in those without (geometric mean $323 \mathrm{kU} /$ litre, 95\% CI of difference 102 to 612 , $\mathrm{p}=0.020$ ) (figure 3 ). The proportion of patients with aeroallergen sensitisation was comparable in those with (97.2\%) and those without AR (91.7\%, $\mathrm{p}=0.20)$; only grass pollen sensitisation differed between these groups (table 2). Patients with AR had a higher median number of aeroallergens to which they were sensitised (median 3, IOR 2-5) than children without AR (median 2, IOR 1-3, $\mathrm{p}=0.33$ ).

\section{Relationship of ACQ scores to allergic rhinitis, adjusted for degree of inflammation or sensitisation}

To establish whether the relationship of AR to ACQ scores was confounded by the degree of lower airway inflammation, or by the severity of allergen sensitisation, we built multiple logistic regression models with $\mathrm{ACQ}$ (dichotomised in scores $<1.0$ (well controlled asthma) or $\geq 1.0$ (incompletely controlled asthma)) as the dependent variable. In these models, the significant association of AR to low ACQ scores remained significant after adjustment for FeNO levels (OR 3.13, 95\% CI 1.10 to 8.85, $\mathrm{p}=0.009$ ), total serum IgE levels (OR 2.89, $95 \%$ CI 1.13 to 7.40 ,

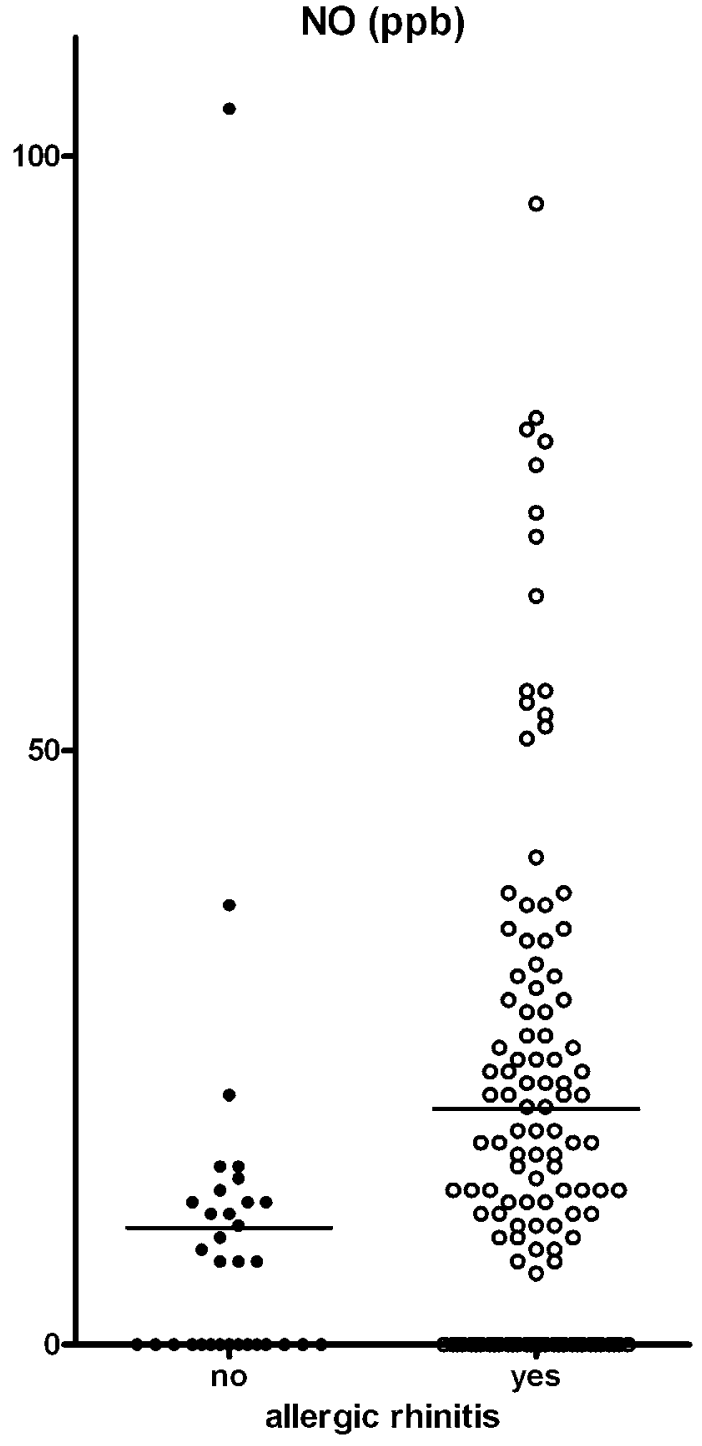

Figure 2 Fraction of nitric oxide in exhaled air (FeNO) values in patients with asthma with and without allergic rhinitis. Horizontal bars represent median values. FeNO levels are significantly higher in patients with asthma and allergic rhinitis than in those without allergic rhinitis $(p=0.001)$. NO, nitric oxide.

$\mathrm{p}=0.028$ ) or the number of aeroallergen sensitisations (OR 2.78, 95\% CI 1.02 to $11.4, \mathrm{p}=0.045$ ).

We also examined whether treatment for AR influenced the association of AR to ACQ scores. The results are presented in table 3. Reported use of rhinitis medication in general did not remove the significant impact of AR on ACQ scores $(p=0.55)$. However, patients who used nasal corticosteroids for AR, either alone or in combination with antihistamines or montelukast, had lower ACQ scores (median 0.58, IOR 0.17-1.17) than those with AR who did not use nasal corticosteroids (median ACQ 1.0, IOR 0.67-1.83), although the difference did not reach statistical significance $(p=0.154)$. After adjusting for use of nasal corticosteroids in a multiple logistic regression model, the relationship of AR to ACQ scores was no longer significant (OR 0.72, 95\% CI 0.47 to $1.12, p=0.150$, table 3 ). In the 157 patients with $A R$ symptoms, however, nasal corticosteroid therapy was not associated with ACQ scores $<1.0$ in a logistic regression model after adjustment for age and gender (OR 1.16, 95\% CI 0.60 to 2.17, $\mathrm{p}=0.664)$. 


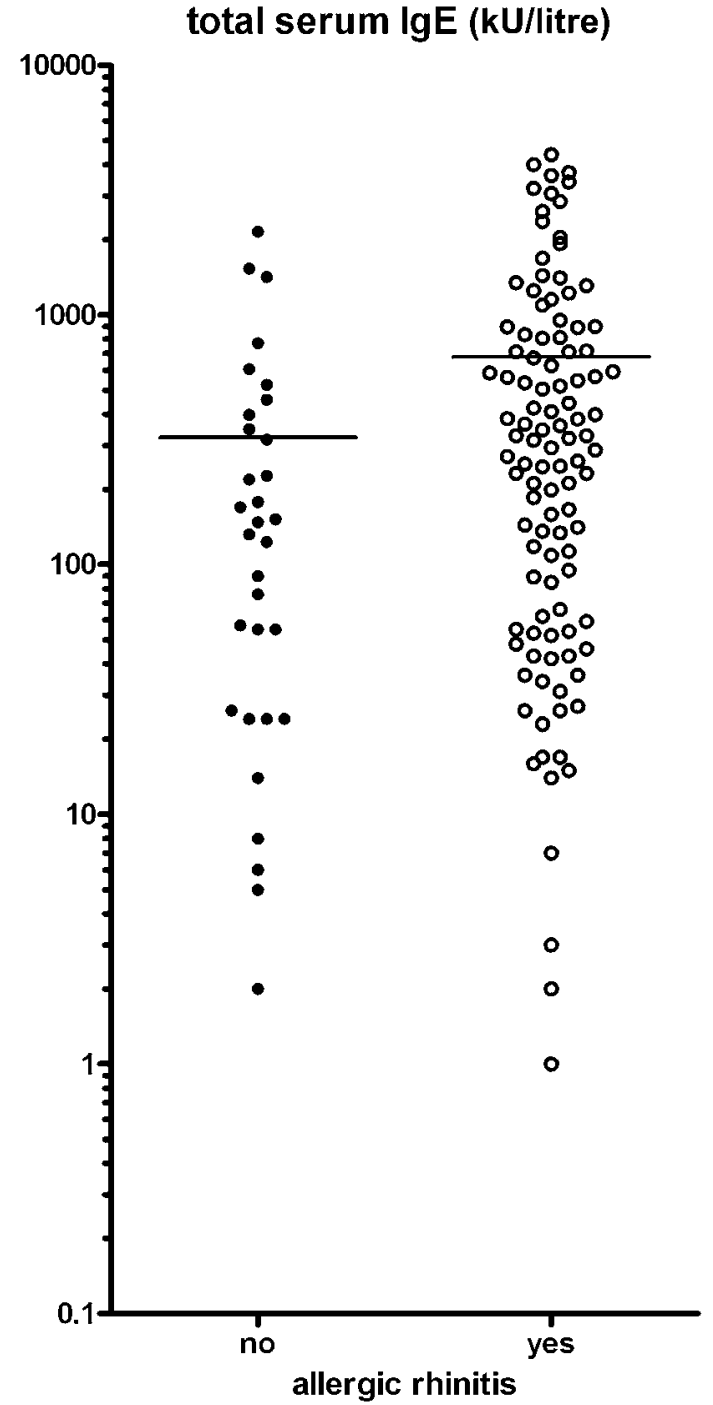

Figure 3 Total serum IgE levels in patients with asthma with and without allergic rhinitis. Horizontal bars represent median values. IgE levels are significantly higher in patients with asthma and allergic rhinitis than in those without allergic rhinitis $(p=0.020)$.

\section{DISCUSSION}

This study shows that AR is a common and relevant comorbid condition in patients with asthma. The large majority $(76 \%)$ of patients with asthma in our study had symptoms of AR, and the presence of AR had a significant influence on asthma control, independent of lower airway inflammation or aeroallergen sensitisation. The association of AR to level of asthma control

Table 2 Number (\%) of patients sensitised to aeroallergens in 131 patients with asthma with and without allergic rhinitis

\begin{tabular}{lccc}
\hline & $\begin{array}{l}\text { Patients with } \\
\text { allergic rhinitis } \\
(\mathbf{n = 1 0 4 )}\end{array}$ & $\begin{array}{l}\text { Patients without } \\
\text { allergic rhinitis } \\
(\mathbf{n = 2 7 )}\end{array}$ & p Value \\
\hline House dust mite & $86(82.7)$ & $21(77.8)$ & 0.556 \\
Cat dander & $58(51.3)$ & $8(30.8)$ & 0.058 \\
Dog dander & $64(56.1)$ & $11(40.7)$ & 0.149 \\
Grass pollen & $62(61.4)$ & $7(26.9)$ & 0.002 \\
Tree pollen & $45(50.6)$ & $5(26.3)$ & 0.054 \\
Any aeroallergen & $105(97.2)$ & $22(91.7)$ & 0.197 \\
\hline
\end{tabular}

Table 3 Association of allergic rhinitis to Asthma Control Questionnaire (ACO) score $<$ or $\geq 1.0$ (indicating well or incompletely controlled asthma, respectively), adjusted for treatment for allergic rhinitis, in 203 patients with asthma with allergic rhinitis symptoms

\begin{tabular}{lll}
\hline & $\mathbf{O R}^{*}$ (95\% CI) & p Value \\
\hline Unadjusted & $2.74(1.28$ to 5.91$)$ & 0.0081 \\
Adjusted for any treatment for allergic rhinitis & $2.41(1.08$ to 5.39$)$ & 0.033 \\
Adjusted for use of oral antihistamines & $2.62(1.22$ to 5.67$)$ & 0.014 \\
Adjusted for use of nasal corticosteroids & $0.72(0.47$ to 1.12$)$ & 0.150 \\
\hline
\end{tabular}

${ }^{*} \mathrm{OR}$ for $\mathrm{ACQ} \geq 1.0$ (incompletely controlled asthma) in patients with allergic rhinitis compared with those without allergic rhinitis.

was not significant in patients who received nasal corticosteroid treatment for AR but remained significant in those not treated for rhinitis symptoms, or those receiving only oral antihistamines or montelukast. This suggests that recognition of AR in patients with asthma, and its prompt treatment with nasal corticosteroids, may improve asthma control in paediatric patients. Randomised clinical trials are required to test this hypothesis.

The rhinitis prevalence of $76 \%$ in this study is comparable to previous studies on AR in children with asthma. ${ }^{3-7}$ Despite this high prevalence, surprisingly few studies have examined the impact of AR on asthma morbidity in children, ${ }^{20}$ and the few results available to date have been contradictory. ${ }^{6}$ Our study is novel in that the main focus of our analysis was the impact of AR on asthma control and daily life activity. Although the complaints had an influence on daily life in almost two-thirds of patients $(64.9 \%)$, almost half $(43.9 \%)$ of patients with AR had not been diagnosed or treated as such by a physician. This may be caused either by underreporting by patients (or their parents) or poor recognition of rhinitis symptoms by doctors. Although the symptoms of AR (runny or stuffed nose without having a cold) may be confused with a cold or flu-like symptoms, the duration of such symptoms ( $>14$ days) should prompt the physician to diagnose AR. ${ }^{9}$ The influence of AR on daily activities in our study was comparable to that reported by others. ${ }^{21}$ In a large survey in the USA, parents of children with AR were more likely to describe physical, mental and emotional, and social problems in their children. ${ }^{22}$

The main finding of our study was that AR had a significant influence on the degree of asthma control, even when asthma was well controlled in the large majority (63\%) of patients participating in our survey. ACQ scores were higher in patients with AR than in those without (figure 1), and ACQ scores $\geq 1.0$ (indicating incompletely controlled asthma ${ }^{16}$ ) were more likely in this group of patients (table 3 ). Although aeroallergen sensitisation tended to be more common in patients with AR than in those without (table 2), the relationship between the presence of $\mathrm{AR}$ and incomplete asthma control remained significant after adjustment for total serum IgE levels or the number of aeroallergens to which the patient was sensitised. We have previously shown that sensitisation to numerous allergens is associated with a more severe clinical atopic phenotype. ${ }^{23}$ Apparently, the relationship of AR to asthma control is independent of this phenomenon of polysensitization.

The significant influence of AR on asthma control suggests that treatment of AR may help to improve asthma morbidity. In a retrospective cohort study in 4944 adult patients, those who were treated for AR had a significantly lower risk (6.6\% vs $1.3 \%$, $\mathrm{p}=0.001$ ) of subsequent asthma-related events such as emergency department visits or hospitalisation than those who were not treated for $\mathrm{AR}^{24}$ Two recent randomised controlled trials in 
adults examined the effect of intranasal steroids on asthma. In one study, although nasal corticosteroid therapy improved nasal inspiratory flow and rhinitis quality of life, there was no inhaled steroid sparing effect of treating AR with nasal corticosteroids. ${ }^{25}$ In the other study, treatment of AR with nasal steroids was associated with a non-significant trend in improving asthma control test scores $(p=0.08) .{ }^{25}{ }^{26}$ No such randomised trials have been performed in children. One study showed that administration of an ICS through a valved holding chamber with facemask improved nasal symptoms but had no influence on asthma symptoms or lung function in children with asthma and AR. ${ }^{27}$ Asthma control was not assessed in this study. Our results suggest that nasal corticosteroid treatment of AR, but not therapy with antihistamines or montelukast, may improve asthma control in children. The significant relationship of AR symptoms to incomplete asthma control disappeared after adjusting for nasal corticosteroid therapy (table 3), although there was no direct relationship between nasal corticosteroid therapy and ACQ score when only the patients with AR were analysed. This may be due to loss of statistical power to detect such a relationship in the smaller subset of patients with AR symptoms. This observation is in line with previous studies showing the superiority of nasal corticosteroids over other treatment modalities in children with AR. ${ }^{9}$ In our study, AR had no impact on pulmonary function in patients with asthma. The overall normal level of lung function in our study group (table 1) is in accordance with a previous report from our clinic. ${ }^{28}$ However, studies in adults have demonstrated a modest improvement in $\mathrm{FEV}_{1}$ (mean $5.45 \%$, 95\% CI $1.95 \%$ to $8.96 \%$, $\mathrm{p}=0.004)$ in patients with asthma after treatment for $A R .^{25}$ Such an effect on pulmonary function is unlikely to be found in children because the room for improvement is too small. Therefore, studies on AR treatment in children with asthma should focus on asthma control and exacerbations as primary endpoints. Although FeNO was higher in patients with AR than in those without (figure 2), the relationship between $A R$ and poor asthma control remained significant after adjustment for FeNO levels, and nasal corticosteroid treatment was associated with lower FeNO levels. Previous studies have shown that a subgroup of children with asthma have persistently high FeNO levels, even when treated with high-dose ICS therapy. ${ }^{29}$ Our observations indicate that treatment with nasal corticosteroids does not alter this. As a result, FeNO is probably not a useful endpoint in studies of nasal corticosteroid effects in children with asthma and AR. The cause of the higher FeNO levels in patients with AR is not entirely clear. Although it is conceivable that allergic inflammation of nasal mucosa could increase local nitric oxide production, and that this may increase nasal contribution to FeNO measurements, the measurement technique for FeNO is intended to avoid nasal contamination. ${ }^{23}$ Therefore, this is unlikely to be the main reason for the higher FeNO levels observed in our patients with AR. Two alternative explanations should be considered. First, it may be argued that patients with asthma and AR have a more severe atopic phenotype, ${ }^{30}{ }^{31}$ and this is reflected by higher FeNO levels. Second, because there is cross-talk between nasal and lower airway mucosa, ${ }^{32} 33$ ongoing nasal inflammation may increase eosinophilic lower airway inflammation and increase FeNO levels.

The main limitation of our study is its cross-sectional nature. Associations found in cross-sectional studies should be interpreted cautiously. Although the results of our study strongly suggest that AR has a significant negative impact on asthma morbidity in children, and that nasal corticosteroid treatment may eliminate this, prospective, preferably randomised controlled studies are needed to confirm this. Second, it may be argued that our definition of AR was non-specific, including all children with rhinitis symptoms outside a cold. Although this definition indeed comprises (IgE-mediated) AR and other causes of chronic rhinitis, AR is usually defined in the same way as in our study, in clinical practice and in research. ${ }^{34} 35$ Finally, we would like to add a cautionary note in generalising our results. Our study was performed in a hospital-based paediatric asthma clinic, in which patients are only seen and followed up when referred by a general practitioner, most commonly because of difficult to control asthma. That this may introduce selection bias is perhaps illustrated by the high prevalence of allergic sensitisation in our cohort (table 2). Further studies are needed to examine the effect of AR and its treatment on asthma control in children in other settings.

In summary, asthma and AR often coexist in children, and the presence of $\mathrm{AR}$ is associated with poorer asthma control, independent of FeNO and allergen sensitisation levels. Despite its impact on daily life activities on asthma control, AR is frequently unrecognised and undertreated. Recognition of AR in children with asthma and adequate treatment with nasal corticosteroids are likely to improve asthma control. This is particularly relevant in children with difficult-to-treat asthma. Randomised controlled trials on the effects of AR treatment on asthma control in children with asthma and AR are urgently needed.

\section{Competing interests None.}

Ethics approval Medical Ethics Committee Isala klinieken Zwolle.

Contributors Eric de Groot is the primary author. Research is conducted by Eric de Groot and Anke Nijkamp. Eric Duiverman and Paul Brand have contributed by extensive editing and advising on the paper.

Provenance and peer review Not commissioned; externally peer reviewed.

\section{REFERENCES}

1. Bousquet J, Khaltaev N, Cruz AA, et al. Allergic rhinitis and its impact on asthma (ARIA) 2008 update (in collaboration with the World Health Organization, GA(2)LEN and AllerGen). Allergy 2008;63(Suppl 86):8-160.

2. Braunstahl GJ. The unified immune system: respiratory tract-nasobronchial interaction mechanisms in allergic airway disease. J Allergy Clin Immunol 2005;115:142-8.

3. Shamssain $\mathbf{M H}$, Shamsian N. Prevalence and severity of asthma, rhinitis, and atopic eczema in 13- to 14-year-old schoolchildren from the northeast of England. Ann Allergy Asthma Immunol 2001;86:428-32.

4. Janahi IA, Bener A, Bush A. Prevalence of asthma among Oatari schoolchildren: International Study of Asthma and Allergies in Childhood, Oatar. Pediatr Pulmonol 2006;41:80-6.

5. Masuda S, Fujisawa T, Katsumata $\mathrm{H}$, et al. High prevalence and young onset of allergic rhinitis in children with bronchial asthma. Pediatr Allergy Immunol 2008;19:517-22.

6. Hamouda S, Karila C, Connault T, et al. Allergic rhinitis in children with asthma: a questionnaire-based study. Clin Exp Allergy 2008;38:761-6.

7. Sanchez-Lerma B, Morales-Chirivella FJ, Penuelas I, et al. High prevalence of asthma and allergic diseases in children aged 6 and 7 years from the Canary Islands: the International Study of Asthma and Allergies in Childhood. J Investig Allergol Clin Immunol 2009;19:383-90.

8. Brozek JL, Bousquet J, Baena-Cagnani CE, et al. Allergic Rhinitis and its Impact on Asthma (ARIA) guidelines: 2010 revision. J Allergy Clin Immunol 2010:126:466-76.

9. de Groot $\mathbf{H}$, Brand PL, Fokkens WF, et al. Allergic rhinoconjunctivitis in children. BMJ 2007;335:985-8

10. Global Initiative for Asthma. Global Strategy for Asthma Management and Prevention. NIH Publication Number 02-3659 1995. Global initiative for asthma, 2008. http://www.ginasthma.org/guidelines-gina-report-global-strategy-for-asthma.html (accessed Dec 2010).

11. Asher MI, Keil U, Anderson HR, et al. International Study of Asthma and Allergies in Childhood (ISAAC): rationale and methods. Eur Respir J 1995:8:483-91.

12. Juniper EF, Guyatt GH, Cox FM, et al. Development and validation of the Mini Asthma Quality of Life questionnaire. Eur Respir J 1999;14:32-8.

13. Juniper EF, Gruffydd-Jones K, Ward S, et al. Asthma Control Questionnaire in children: validation, measurement properties, interpretation. Eur Respir J 2010;36:1410-16.

14. Kuethe MC, Vaessen-Verberne AA, Bindels PJ, et al. Children with asthma on inhaled corticosteroids managed in general practice or by hospital paediatricians: is there a difference? Prim Care Respir J 2010;19:62-7, 8p. 
15. van Dellen OM, Stronks K, Bindels PJ, et al. Predictors of asthma control in children from different ethnic origins living in Amsterdam. Respir Med 2007:101:779-85.

16. Juniper EF, Bousquet J, Abetz L, et al. Identifying 'well-controlled' and 'not well-controlled' asthma using the Asthma Control Questionnaire. Respir Med 2006;100:616-21.

17. Quanjer PH, Tammeling GJ, Cotes JE, et al. Lung volumes and forced ventilatory flows. Report Working Party Standardization of Lung Function Tests, European Community for Steel and Coal. Official Statement of the European Respiratory Society. Eur Respir J Suppl 1993;16:5-40.

18. Stanojevic S, Wade A, Stocks J, et al. Reference ranges for spirometry across all ages: a new approach. Am J Respir Crit Care Med 2008;177:253-60.

19. American Thoracic Society European Respiratory Society. ATS/ERS recommendations for standardised procedures for the online and offline measurement of exhaled lower respiratory nitric oxide and nasal nitric oxide, 2005. Am J Respir Crit Care Med 2005;171:912-30.

20. de Groot EP, Duiverman EJ, Brand PL. Comorbidities of asthma during childhood: possibly important, yet poorly studied. Eur Respir J 2010;36:671-8.

21. Kocabas CN, Civelek E, Sackesen C, et al. Burden of rhinitis in children with asthma. Pediatr Pulmonol 2005; 40:235-40.

22. Meltzer EO, Blaiss MS, Derebery MJ, et al. Burden of allergic rhinitis: results from the Pediatric Allergies in America survey. J Allergy Clin Immunol 2009:124:S43-70.

23. de Jong $\mathbf{A B}$, Dikkeschei $L D$, Brand PL. Sensitization patterns to food and inhalant allergens in childhood: a comparison of non-sensitised, monosensitized, and polysensitized children. Pediatr Allergy Immunol 2011;22:166-71.

24. Crystal-Peters J, Neslusan C, Crown WH, et al. Treating allergic rhinitis in patients with comorbid asthma: the risk of asthma-related hospitalizations and emergency department visits. J Allergy Clin Immunol 2002;109:57-62.

25. Nair A, Vaidyanathan $\mathrm{S}$, Clearie $\mathrm{K}$, et al. Steroid sparing effects of intranasal corticosteroids in asthma and allergic rhinitis. Allergy 2010;65:359-67.
26. Scichilone N, Arrigo R, Paterno A, et al. The effect of intranasal corticosteroids on asthma control and quality of life in allergic rhinitis with mild asthma. J Asthma 2011:48:41-7.

27. Camargos $\mathbf{P}$, Ibiapina $\mathrm{C}$, Lasmar L, et al. Obtaining concomitant control of allergic rhinitis and asthma with a nasally inhaled corticosteroid. Allergy 2007:62:310-16

28. Baatenburg de JA, Brouwer AF, Roorda RJ, et al. Normal lung function in children with mild to moderate persistent asthma well controlled by inhaled corticosteroids. J Allergy Clin Immunol 2006;118:280-2.

29. Pijnenburg MW, Bakker EM, Lever S, et al. High fractional concentration of nitric oxide in exhaled air despite steroid treatment in asthmatic children. Clin Exp Allergy 2005:35:920-5.

30. Moore WC, Meyers DA, Wenzel SE, et al. Identification of asthma phenotypes using cluster analysis in the Severe Asthma Research Program. Am J Respir Crit Care Med 2010;181:315-23.

31. Sears MR, Greene JM, Willan AR, et al. A longitudinal, population-based, cohort study of childhood asthma followed to adulthood. N Engl J Med 2003;349:1414-22.

32. Bousquet J, Vignola AM, Demoly P. Links between rhinitis and asthma. Allergy 2003:58:691-706.

33. Togias A. Systemic cross-talk between the lung and the nose. Am J Respir Crit Care Med 2001;164:726-7.

34. Peroni DG, Piacentini GL, Alfonsi L, et al. Rhinitis in pre-school children: prevalence, association with allergic diseases and risk factors. Clin Exp Allergy 2003;33:1349-54.

35. Weinmayr G, Forastiere F, Weiland SK, et al. International variation in prevalence of rhinitis and its relationship with sensitisation to perennial and seasonal allergens. Eur Respir J 2008:32:1250-61.

\section{DIFFERENTIAL DIAGNOSIS}

\section{Trustworthy guidance on your iPhone}
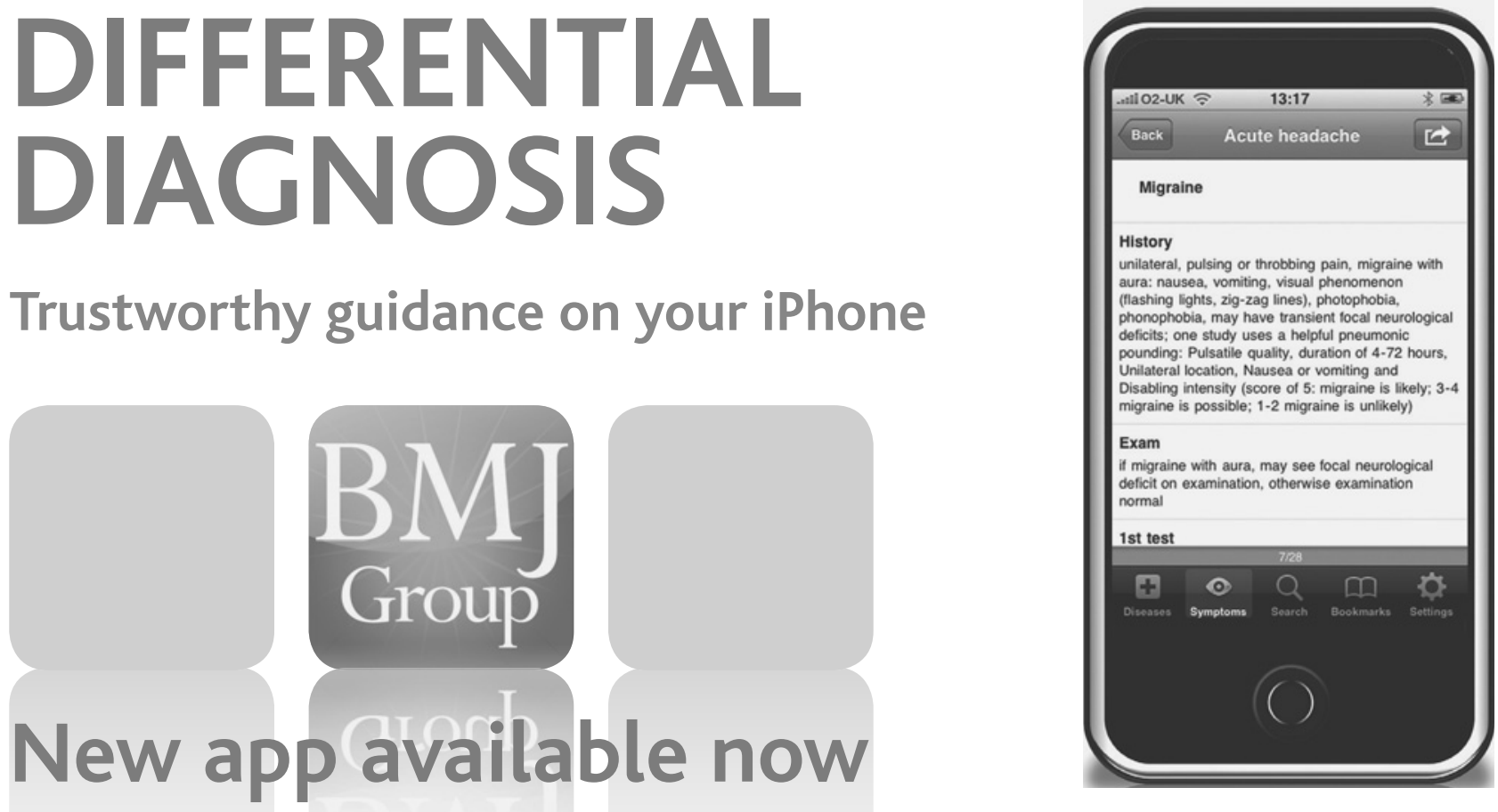

\section{Find out more at bestpractice.bmj.com/differentials}

